\title{
Accesibilidad en el ámbito de la salud materna de mujeres rurales de tres localidades del norte argentino'
}

\section{Rural women's accessibly to maternal health care in three villages located in the northern region of Argentina}

\author{
Fernando Landini \\ Consejo Nacional de Investigaciones Científicas y Técnicas. Ciudad \\ Autónoma de Buenos Aires, Argentina. \\ Universidad de la Cuenca del Plata. Instituto de Investigaciones \\ Científicas. Posadas, Misiones, Argentina. \\ Universidad de Morón. Facultad de Agronomía y Ciencias \\ Agroalimentarias. Buenos Aires, Argentina. \\ E-mail: landini_ferळhotmail.com

\section{Valeria González Cowes} \\ Universidad de Buenos Aires. Facultad de Psicología. Cátedra \\ Psicología Social I. Ciudad Autónoma de Buenos Aires, Argentina. \\ Ministerio de Salud de la Nación. Comisión Nacional Salud \\ Investiga. Buenos Aires, Argentina. \\ E-mail: valeria.gonzalezcowesळgmail.com

\section{Vanina Bianqui} \\ Universidad de Buenos Aires. Facultad de Psicología. Cátedra \\ Estrategias de Intervención Comunitaria. Ciudad Autónoma de \\ Buenos Aires, Argentina. \\ Universidad de Morón. Facultad de Filosofía, Ciencias de la \\ Educación y Humanidades. Cátedra de Psicología Forense. Buenos \\ Aires, Argentina. \\ Consejo Nacional de Investigaciones Científicas y Técnicas. Ciudad \\ Autónoma de Buenos Aires, Argentina. \\ E-mail: vbianqui84@hotmail.com
}

\author{
Sabrina Logiovine \\ Ministerio de Salud de la Nación. Comisión Nacional Salud \\ Investiga. Ciudad Autónoma de Buenos Aires, Argentina. \\ Consejo Nacional de Investigaciones Científicas y Técnicas. Ciudad \\ Autónoma de Buenos Aires, Argentina. \\ E-mail: sabrina_logœhotmail.com \\ Jessica Vázquez \\ Ministerio de Salud de la Nación. Comisión Nacional Salud \\ Investiga. Ciudad Autónoma de Buenos Aires, Argentina. \\ E-mail: jessicavazquez77@hotmail.com

\section{Sergio Viudes} \\ Universidad de la Cuenca del Plata. Facultad de Psicología, \\ Educación y Relaciones Humanas. Posadas, Misiones, Argentina. \\ Ministerio de Salud de la Nación. Comisión Nacional Salud \\ Investiga. Ciudad Autónoma de Buenos Aires, Argentina. \\ E-mail: sgviudesळgmail.com
}

\section{Correspondencia \\ Fernando Landini \\ Terrero 1165 , 3A. Ciudad Autónoma de Buenos Aires, Argentina. Código Postal 1416.}

1 Fuente de financiamiento de la investigación: Comisión Nacional Salud Investiga. Ministerio de Salud de la Nación, Argentina. 


\section{Resumen}

Si bien las mujeres rurales poseen mayor riesgo de morir por causas relacionadas con el embarazo, el parto y puerperio, los estudios que abordan el tema suelen prestar escasa atención a la variable 'ruralidad'. Se realizó un estudio comparativo de tres casos apoyado en un abordaje cualitativo orientado a identificar, describir y analizar las barreras de orden geográfico, económico y administrativo que limitan el acceso de mujeres rurales al sistema público de salud en el ámbito de la salud materna. Se seleccionaron tres territorios de diferentes provincias del norte argentino: el municipio de Goya (provincia de Corrientes), el municipio de Dos Arroyos (provincia de Misiones) y la comuna de Ranchillos (provincia de Tucumán). En cada caso se realizaron 15 entrevistas, en su mayoría a mujeres rurales y a profesionales que atienden en el ámbito de la salud materna. Las entrevistas fueron transcriptas y los textos categorizados a partir de ejes temáticos expresados en los objetivos de la investigación. Se concluye que existen importantes barreras de accesibilidad que articulan problemas de nivel geográfico, económico y administrativo en ámbitos rurales.

Palabras clave: Accesibilidad a los Servicios de Salud; Servicios de Salud Materna; Población Rural; Salud de la Mujer; Mortalidad Materna.

\section{Abstract}

Despite rural women's increased risk of dying due to pregnancy, childbirth and puerperium related causes, investigations usually only pay scarce attention to the variable 'rurality'. Thus, we conducted a qualitative research comparing three case studies aiming to identify, describe and analyse geographical, economic and administrative barriers that limit the access of rural women to public maternal health care. Three territories located in different provinces in the northern region of Argentina were selected: the municipality of Goya (province of Corrientes), the municipality of Dos Arroyos (province of Misiones) and the commune of Ranchillos (province of Tucumán). Fifteen interviews were carried out in each case study, mainly with rural women and physicians that provide maternal health care to them. The interviews were typed and their text categorized using the topics expressed in the objectives of this research. We conclude that there are a set of interrelated geographical, economic and administrative barriers limiting maternal health care accessibility in rural areas.

Keywords: Health Services Accessibility; Maternal Health Services; Rural Population; Women's Health; Maternal Mortality. 


\section{Introducción}

En este trabajo se estudia la accesibilidad geográfica, económica y administrativa de mujeres de sectores populares que viven en localidades rurales de tres provincias del norte argentino al sistema sanitario en el ámbito de la salud materna. Las localidades fueron seleccionadas siguiendo criterios de conveniencia, fundamentalmente por la existencia de vínculos de los investigadores con los territorios a estudiar. En concreto, se trabajó en los municipios de Dos Arroyos (provincia de Misiones) y de Goya (provincia de Corrientes), y en la comuna de Ranchillos (provincia de Tucumán). La salud materno-infantil constituye una prioridad a nivel de las políticas públicas de salud en Argentina. No obstante, pese a la atención brindada a la temática, las estadísticas muestran que la mortalidad materna resulta mucho más difícil de reducir que la infantil (Ramírez; Huerto, 2008).

La mortalidad materna se encuentra asociada con mayores niveles de pobreza (Buchbinder, 2008; Houweling et al., 2007). El 99\% de las muertes maternas se producen hoy en los países en desarrollo (Ramírez; Huerto, 2008). A la vez, también se ha informado mayor mortalidad materna en ámbitos rurales (ONU, 2010). Así, la población rural femenina de bajos ingresos, aparece como un sector social particularmente golpeado por la mortalidad materna. Pese a esto, en Argentina no existen datos oficiales que indiquen la procedencia urbana o rural de las mujeres que mueren por causas asociadas a la maternidad. A la vez, en Latinoamérica son contados los estudios que abordan las especificidades rurales de la mortalidad materna, a diferencia de lo que sucede en estudios provenientes de África o del sudeste asiático, donde la ruralidad es tomada como variable fundamental a la hora de indagar sobre mortalidad materna (Adamson et al., 2012; Hounton; Byass; Brahima, 2009; Igberase; Ebeigbe, 2007; Iyengar et al., 2009). En la bibliografía referida a mortalidad materno-infantil pueden distinguirse tres tipos de estudios. Primero, trabajos abocados a la producción de estadísticas, incluyendo estadísticas generales en torno a morbilidad y mortalidad materno-infantil o sobre variables de carácter socio demográfico o de perfil médico asociadas (Buchbinder, 2008; Echarri, 2004; Uriburu et al., 2007). El segundo grupo aborda problemas médicos asociados a la salud/enfermedad materno-infantil (Hartley et al., 2011; Rodríguez, 2005). Por último, también existe bibliografía orientada a la evaluación de programas, políticas o instituciones en el área de la salud materno-infantil (Macias, 2009; Rojo et al. 2006). También existen trabajos vinculados con otras temáticas o realizados desde otros enfoques. No obstante, a nivel general parece existir una tendencia a abordar la salud materna desde una perspectiva que pone el énfasis, por un lado, en la patología (incluida la construcción de datos sobre morbilidad y mortalidad) y, por el otro, en las políticas o proyectos que se implementan en relación a ella. Por el contrario, en este trabajo nos proponemos pensar la salud materna desde una mirada diferente: desde la accesibilidad de las mujeres rurales al sistema de salud.

La accesibilidad de la población al sistema de salud constituye uno de los pilares de la Atención Primaria de la Salud y una de las principales preocupaciones de las políticas sanitarias (Vargas-Lorenzo; Vazquez-Navarrete; Mogollon-Perez, 2010). La accesibilidad ha sido definida de diferentes maneras (Henderson; Kendall, 2011; Moreno et al., 2009; Otalvaro; Arango, 2009; Travassos; Martins, 2004). La mayor parte de los autores tiende a pensarla en términos del ajuste entre las características de los servicios y sus profesionales, y las necesidades, situaciones o particularidades de los beneficiarios (Arakawa et al., 2011; Pinho; Hernandez; Kantorski, 2009; Gaioso; Mishima, 2007; Clementino; Miranda, 2010). Por ejemplo, Martins et al. (2010) la han definido como "la posibilidad de uso de los sistemas de salud cuando son necesarios, lo que se expresa en las características de la oferta que facilitan u obstaculizan la posibilidad de la gente de usar los servicios de salud cuando los necesitan" (p. 439). Respecto de la ruralidad, existe consenso en la existencia de una desventaja para las poblaciones rurales frente a las urbanas debido a las distancias y a los costos de transporte, entre otros factores (Galambros, 2005). Específicamente, respecto de la accesibilidad por razones de salud materna en ámbitos rurales, diferentes estudios también han reportado esta situación de desventaja (Grzybowski; Stoll; Kornelsen, 2011; Kesterton et al., 2010; Matthews et al., 2010; Yakong et al., 2010), lo que se traduce en mayor riesgo de mortalidad materna. 
Existen dos tipologías ampliamente utilizadas para caracterizar la accesibilidad (Landini; Cowes; D’Amore, 2014). En este estudio utilizamos la que diferencia entre accesibilidad geográfica, financiera y administrativa (Arakawa et al., 2011; Barbastefano; Girianelli; Vargens, 2010). Siguiendo a diversos autores (Comes et al., 2007; Comes; Stolkiner, 2005) entendemos a la accesibilidad geográfica como aquella que se relaciona con la distancia entre los beneficiarios y los servicios, a la económica como la que focaliza en la capacidad financiera de las personas para cubrir los costos vinculados con la atención, y la administrativa como aquella relacionada con las trabas burocráticas para acceder al servicio.

En resumen, en este trabajo nos ocuparemos de pensar la salud materna de un sector de la población particularmente vulnerable: las mujeres rurales de sectores de bajos ingresos. Para hacerlo, pondremos el foco en la accesibilidad de estas mujeres al sistema de salud. En concreto, nos ocuparemos de identificar, describir y analizar las barreras de orden geográfico, económico y administrativo que limitan su acceso al sistema de salud en el ámbito de la salud materna de mujeres de tres localidades rurales del norte argentino.

\section{Metodología}

Se llevó adelante un estudio comparativo de casos de carácter transversal. El encuadre de la investigación fue cualitativo, lo que llevó a poner el foco en la reconstrucción de las comprensiones de los participantes, particularmente pobladoras rurales y profesionales de la salud que trabajan en el ámbito de la salud materna, en tres diferentes territorios del norte argentino. También se entrevistó a otros actores cuando se lo consideró relevante, incluyendo autoridades hospitalarias, autoridades municipales, agentes de salud y referentes comunitarios. En cada uno de los tres estudios de caso se realizaron 15 entrevistas semi estructuradas. Dentro de cada uno de los tres territorios elegidos para la investigación se identificaron áreas rurales específicas para realizar las entrevistas a las pobladoras. En Goya (Corrientes) se seleccionaron los parajes de Santa Rita y San Juan, en Dos Arroyos (Misiones) la zona de Cerro López y en Ranchillos (Tucumán), áreas rurales indiferenciadas de la comuna. También se realizaron entrevistas a profesionales de la salud que trabajan en el ámbito de la salud materna con mujeres rurales y a otros actores que contextualmente resultaron relevantes. En este caso, las entrevistas se llevaron a cabo tanto en las localidades rurales seleccionadas como en las cabeceras de los tres distritos. En Goya se entrevistó a 10 mujeres rurales y a 5 profesionales. En Dos Arroyos a 8 mujeres rurales, a 3 profesionales y a otros 4 actores. En Ranchillos a 9 mujeres rurales, a 4 profesionales y a otros 2 actores. Las mujeres fueron seleccionadas por estar embarazadas o tener hijos de hasta 5 años de edad, residir en las áreas rurales seleccionadas y pertenecer a sectores de bajos ingresos (al ser de familias de agricultores familiares o por no superar su ingreso familiar el salario mínimo en más de un 50\%). Para identificar a estas mujeres se contó con el apoyo de profesionales o agentes sanitarios del lugar. Para el caso de los profesionales se estableció como criterio de inclusión en la muestra atender población rural de las localidades en cuestión en relación a problemas de salud materna y tener al menos 6 meses de experiencia en la tarea. Antes de cada entrevista se procedió a solicitar un consentimiento informado verbal. A la vez, para respetar la confidencialidad de los participantes, en este texto se evitan nombres o se los modifica cuando se los usa. Para analizar los resultados se procedió a transcribir las entrevistas y a categorizarlas con el software Atlas Ti. Los ejes de categorización fundamentales corresponden a los subtítulos del siguiente apartado incluyendo 'organización del sistema de salud', 'problemas de accesibilidad geográfica, económica y administrativa' y 'estrategias de los profesionales'. En los resultados, para identificar a los encuestados se utilizará el siguiente código: P referirá a 'Profesionales', M a 'Mujeres', G a 'Goya', D a 'Dos Arroyos' y R a 'Ranchillos'. Así, por ejemplo, PR significará: 'profesional de Ranchillos'.

\section{Resultados y discusión}

\section{Las localidades visitadas y la organización de sus sistemas de salud}

El municipio de Goya se encuentra en el suroeste de la provincia de Corrientes. Posee una ciudad cabecera, Goya, y un conjunto de parajes rurales. Se realizaron entrevistas en dos de ellos, Santa Rita y 
San Juan. El primero se encuentra a 73 km de Goya, 7 por camino de tierra hasta la ruta 12 y luego 66 $\mathrm{km}$ hasta la ciudad. El segundo se ubica junto a la ruta a $60 \mathrm{~km}$ de Goya. La ciudad cabecera cuenta con varios Centros de Atención Primaria de la Salud (CAPS) y con dos Centros Integradores Comunitarios (CIC). Dispone además de un Hospital Regional de segundo nivel. En la zona rural hay 4 Salas de Atención Primaria de la Salud (SAPS) ubicadas en diferentes parajes, las cuales generalmente cuentan con la presencia de un agente sanitario. En ellas, cada 7 o 14 días se realizan operativos rurales de salud llevados adelante por la Municipalidad y por el Instituto Provincial del Tabaco. En los parajes que no disponen de SAPS la periodicidad es mensual o incluso más espaciada. Las pobladoras rurales del municipio pueden iniciar controles prenatales en los operativos rurales. Allí reciben un primer chequeo clínico. No obstante, los profesionales no proponen el seguimiento en el contexto de los operativos, sino que indican estudios y derivan para atención en la ciudad de Goya, cabecera municipal. Así, la atención continúa en el CAPS que funciona en el edificio de la Municipalidad, en alguno de los CIC o directamente en el hospital regional de Goya, que es donde se realizan partos.

En Misiones, el trabajo fue realizado en el paraje Cerro López, municipio de Dos Arroyos, departamento de Alem. El hospital de segundo nivel que corresponde a la zona está ubicado en Alem, distante 25 $\mathrm{km}$ del paraje (entre 1 y $10 \mathrm{~km}$ por camino de tierra, según la ubicación de la vivienda, mas $20 \mathrm{~km}$ por ruta asfaltada). El trayecto de tierra a la ruta suele hacerse en moto o a pie. Al llegar a la ruta, el traslado a Alem puede realizarse en trasporte público. En las cercanías del paraje existen tres CAPS. El más cercano a Cerro López es 'Km 26', ubicado sobre la ruta a unos $5 \mathrm{~km}$ del paraje. Si bien en Cerro López técnicamente también existe un CAPS, parecería más apropiado definirlo como una Posta Sanitaria, dado que allí no hay ni consultorio ni médico que visite el lugar, aunque sí hay un agente sanitario algunos días de la semana. En Alem también hay dos instituciones privadas que realizan partos, a las cuales concurre un porcentaje importante de la población rural, ya que muchos poseen la obra social de la Asociación de Plantadores de Tabaco de Misiones.
La atención en los CAPS se encuentra orientada a la salud materno-infantil. En aquellos ubicados en la zona de Cerro López se presta atención médica uno, dos o tres días por semana, dependiendo del caso. Allí las mujeres pueden controlar su embarazo con un profesional, pero deben ir a Alem para hacerse estudios complementarios como análisis de sangre y ecografías.

En Tucumán el estudio fue realizado el área rural de Ranchillos. El pueblo cabecera de Ranchillos se encuentra a $24 \mathrm{~km}$ de la capital provincial. En esta última existen distintos efectores de tercer nivel, incluyendo una Maternidad. El hospital de Ranchillos, de segundo nivel, es cabecera del área operativa, en la cual también existen ocho CAPS ubicados en zonas rurales, junto con un CIC en Ranchillos. La distancia de las comunidades rurales a los CAPS y de éstos al hospital de Ranchillos es variable. La distancia máxima de las poblaciones rurales de Ranchillos a los CAPS es de 6 ó $7 \mathrm{~km}$ aproximadamente, y de los CAPS al Hospital de Ranchillos de entre 6 y $22 \mathrm{~km}$. La distancia de los CAPS a Ranchillos en algunos casos refiere a ruta asfaltada y en otros (particularmente aquellos más lejanos) a caminos de tierra. Para llegar a los CAPS, las mujeres se acercan a pie, en bicicleta, moto, autobús o auto. Para ir a Ranchillos o a la capital, quienes viven sobre la ruta tienen autobuses cada 30 minutos o una hora. Quienes viven lejos de ella en los distintos caminos vecinales también tienen transporte pero con menor frecuencia.

En la mayoría de los CAPS hay médicos de distintas especialidades (generalista, clínico, pediatra, etc.) y enfermeros que realizan controles y seguimiento de embarazo y puerperio. En general, los médicos de los CAPS trabajan allí todos los días (no circulan por los CAPS de la zona) lo que permite la construcción de un vínculo personal entre profesionales y vecinas. De todas maneras, también hay casos de CAPS que no cuentan con médicos. Estudios complementarios y consultas con otros especialistas se realizan mayormente en el hospital Ranchillos. Allí se realiza seguimiento de embarazo y puerperio, así como estudios de sangre y Papanicolaou (PAP), pero no ecografías. La mayoría de los partos se realizan en la capital provincial (en la Maternidad Nuestra Señora de las Mercedes y en el Hospital 
Avellaneda). No obstante, en situaciones de urgencia también hay partos en Ranchillos. Los CAPS visitados también cuentan con agentes sanitarios. Si bien las actividades realizadas varían mucho, generalmente se orientan a visitar a los pobladores de la zona que se encuentran en situación de mayor vulnerabilidad, incentivando el acercamiento al sistema de salud.

\section{Barreras geográficas y económicas}

Para evaluar la accesibilidad geográfica es necesario considerar tanto la distancia a los lugares donde se brindan las prestaciones como la existencia de transportes y las características de los caminos. Primero, en todos los casos la distancia del lugar donde viven las mujeres a los centros de atención constituye un problema. Un profesional de Groya comenta: "hay parajes que son lejísimos" (PG). En Cerro López una mujer señala: "para que llegue de acá hasta el médico, si es algo grave, te morís en el camino, porque mirá cuanto tenés para salir a la ruta [por tierra] 6 kilómetros” (MD). A la vez, en Ranchillos se planteó que "el hospital me queda lejos" (MR). Más allá de esta coincidencia, un análisis más minucioso muestra una importante diversidad entre casos. En primer lugar, es necesario tomar en cuenta las características de los caminos a recorrer, ya que no es lo mismo residir en las cercanías de la ruta que tener que recorrer $6 \mathrm{~km}$ por camino de tierra o más (que puede ser desde camino consolidado hasta camino vecinal en mal estado). Un profesional de Goya comenta: "si llueve y hay barro no se puede entrar con los vehículos para ir a hacer los operativos". Y agrega: "hay veces que no nos podemos acercar hasta esos lugares o viceversa, ellos hasta nosotros" (PG). En Ranchillos pareciera que el estado de los caminos permite trasladarse aun luego de algo de lluvia: "hay gente que vive en colonia adentro, que está a un kilómetro de acá, que tiene que salir 40 minutos antes con lluvia” (PR). De todas formas, en época de lluvias: "camino de tierra, si es enero, febrero, marzo, que es tiempo de llover, camino intransitable" (Agente Sanitario, Ranchillos). A la vez, también es relevante la existencia de transporte que pase por las zonas rurales, como sucede en Ranchillos, así como su frecuencia; y luego la existencia y características del transporte una vez que se llega a la ruta.
Todos estos elementos marcan una importante diferencia entre los casos. En el paraje Santa Rita (Goya), es necesario recorrer $7 \mathrm{~km}$ de tierra para llegar a la ruta. Este no está muy consolidado, es bastante vulnerable en caso de lluvias y no cuenta con transporte público. Las opciones para llegar a la ruta son ir en moto, a pie, o contratando un vehículo (si se tiene el dinero). Una vez en la ruta hay que recorrer $66 \mathrm{~km}$ para llegar a Goya, con autobuses que pasan cada 3 ó 4 horas. Cerro López (Dos Arroyos) se encuentra a entre 1 y $10 \mathrm{~km}$ de la ruta por camino de tierra, siendo la distancia por ruta a Alem de sólo $20 \mathrm{~km}$, lo que marca la diferencia con Santa Rita. No obstante, al igual que allí, el camino de tierra para llegar a la ruta también es vulnerable a las lluvias y no cuenta con transporte público. Una vez en la ruta, la frecuencia de autobuses a Alem sería algo más alta que en Goya, con trasportes cada tres horas o menos. Ranchillos muestra algunas diferencias notables respecto de los casos anteriores. Como las entrevistas se realizaron en una zona más amplia, encontramos mujeres que viven a una distancia de entre 1 y $5 \mathrm{~km}$ de la ruta, a donde tienen que llegar por camino de tierra. No obstante, allí existe transporte público rural, lo que permite desplazarse con mucho menos esfuerzo, pudiendo llegar a la capital provincial sin tener que hacer transbordo, como sí es necesario en algunos casos para llegar a Ranchillos. La frecuencia del transporte varía según la zona, pudiendo llegar a un mínimo de dos o tres por día. Ya en la ruta es posible encontrar transporte cada hora, o cada media hora incluso. Dados los micros que entran a la zona, los caminos rurales se observan más consolidados, siendo menos vulnerables a las lluvias. Luego de haber llegado a la ruta, el trayecto a la capital provincial será de entre 20 y $30 \mathrm{~km}$ y a Ranchillos de 10 km o menos.

Respecto de la distribución territorial de las prestaciones, en el caso de los parajes estudiados de Goya, las pobladoras deben ir a la ciudad (distante entre $60 \mathrm{~km}$ y $77 \mathrm{~km}$ ) tanto para hacerse estudios complementarios (análisis de laboratorio, ecografía, etc.) como para dar seguimiento clínico a su embarazo. En Cerro López (Dos Arroyos) y en Ranchillos la situación es diferente, ya que es posible hacer seguimiento del embarazo en los CAPS ubicados en las zonas rurales. En Cerro López, los médicos 
están presentes en los CAPS aledaños de uno a tres días por semana, teniendo que realizarse los estudios complementarios en Alem. En Ranchillos la presencia de los profesionales en los CAPS es aun más frecuente. Respecto de los estudios recomendados, la mayoría de ellos (análisis de sangre u orina, PAP) pueden hacerse en el hospital de Ranchillos, con excepción de las ecografías, que deben hacerse o en la capital o en el algún servicio privado en el mismo pueblo.

Para completar la descripción de la accesibilidad geográfica hay que mencionar dos facilitadores. Primero, la disponibilidad de ambulancias para el traslado de emergencias, la cual acorta las 'distancias'. Comentan diferentes entrevistados: "la ambulancia hubo que llamar y vienen" (MG), "llamé a la ambulancia, subimos, fuimos a Alem [...], llegamos justito" (PD). Respecto de las ambulancias no fueron identificadas diferencias entre casos. Segundo, un actor que también puede, en cierto sentido, acortar las distancias entre el sistema de salud y las mujeres son los agentes sanitarios (AS). En Goya, son pocos los AS que están en los territorios. Los que hay, en general están en las SAPS o acompañan los operativos rurales, pero no realizan seguimiento personalizado de las mujeres ni visitan viviendas. En contraste, tanto en Dos Arroyos como en Ranchillos la presencia de AS en los territorios es fuerte, ocupándose tanto de actividades en los mismos CAPS como de visitar y atender familias en situación de vulnerabilidad. Dice un médico en Ranchillos: "el mayor facilitador [de la atención] es la Juana [AS] que las obliga [risas]" (PR). El mismo médico explica que "la atención, fundamentalmente de las embarazadas, empieza por el agente sanitario. Ella capta las embarazadas [...] a veces sabe ella antes que su papá que está embarazada" (PR). En Goya los profesionales también mencionan la existencia de un laboratorio que puede movilizarse por ruta asfaltada para hacer diferentes estudios. No obstante, como no ha sido mencionado por ninguna mujer entrevistada, no será incorporado en análisis posteriores.

Articulada con la accesibilidad geográfica debe mencionarse a la accesibilidad económica. En Goya, las grandes distancias, la ausencia de transporte público en zona rural y la centralización de la atención en la ciudad hacen de la disponibilidad de dinero un elemento crítico. Cuando se le preguntó a una mujer si se había hecho controles en su primer embarazo respondió "no, no me hice porque no tenía plata” (MG). Además, muchas embarazadas también tienen hijos pequeños que no tienen con quién dejar en sus casas, lo que hace que tengan que llevarlos, aumentando así el costo monetario del transporte. La situación es incluso más complicada cuando a la dificultad del transporte se suman problemas para la obtención de turnos, lo que lleva a algunas mujeres a atenderse en un efector privado para no tener que volver otro día: “a veces no me atiende en el día y tengo que esperar al otro día, por eso yo no me voy al hospital. Entonces me voy, pago y ya me atiende, no estoy esperando" (MG). Claro, quien no dispone de dinero simplemente decidirá no hacerse los estudios o asistir a controles. En Cerro López (Dos Arroyos) también aparece el problema del dinero: "en ese tiempo [...] sin tener la plata para pagar un viaje, y ese nos dificultaba un montón" (MD). No obstante, en este caso el problema económico se menciona con mucha menor frecuencia, lo que resulta razonable si se piensa que la posibilidad de atención no se encuentra concentrada en la ciudad y que las distancias son menores. En Ranchillos, la cuestión económica también es muy mencionada, aunque su incidencia no parece ser tan radical como en Goya. Aquí se menciona la dificultad general del dinero para el pago del autobús: "tenía que viajar y a veces no tenía para el boleto y bueno, ya no me iba" (MR). A la vez, aparece la posibilidad de acercar distancias si se puede pagar las ecografías y hacerlas en Ranchillos en el ámbito privado en lugar de viajar a la capital. Dice una profesional "tenemos gran dificultad con las ecografías, no tenemos ecografías gratuitas en el hospital [de Ranchillos], son \$8o que tienen que tener si se las hacen acá" (PR). Caso contrario, deben ir a la capital provincial. En resumen, las barreras económicas se articulan con las geográficas y con la organización del sistema de salud, siendo difícil decir que tienen una incidencia independiente.

\section{Barreras administrativas}

En nuestra investigación hemos encontrado una barrera administrativa fundamental, la obtención de turnos, y otras que poseen menor presencia cuan- 
titativa, aunque no por eso son menos importantes. Para abordar el tema de los turnos, es necesario diferenciar según el objetivo del turno, ya sea para atención médica o realización de estudios, y el lugar donde se recibirá la atención (CAPS, CIC, Hospital, etc.). En las entrevistas, los problemas para la obtención de turnos han sido mencionados insistentemente. En Goya una profesional comenta: "ella [una embarazada] salía del campo, hizo dos intentos de hacer control en el hospital y no logró turno" (PG). Dos mujeres también comentan sus dificultades: "se te hace difícil por el tema del turno, ir allá, volver, ir de nuevo" (MD), "se junta mucha gente y a veces dan 10 números nomás y ya no dan más” (MR). La gestión de turnos es bastante similar en los tres casos estudiados, aunque con algunas diferencias. Para atenderse en los CAPS rurales hay que pedir turno en el día (no se puede pedir con anticipación). Hay que llegar al lugar antes de un horario determinado que suele ser temprano durante la mañana. Una pobladora de Ranchillos explica: "yo me tengo que levantar temprano para ir a sacar número, saco turno y ahí me atiende" (MR). En Goya es diferente porque se trata de operativos rurales, donde las personas son atendidas por orden de llegada, aunque en el fondo es la misma lógica. En ninguno de los casos se han indicado problemas para conseguir estos turnos, lo que sí sucede en otros niveles. Aquí el inconveniente principal es la permanencia de los profesionales en las zonas rurales. En Ranchillos en su mayoría atienden 5 días por semana, en Dos Arroyos de 1 a 3 días por semana según el CAPS, y en Goya con operativos rurales cada 15 días, un mes, o incluso más espaciados.

En los tres estudios de caso, para recibir atención médica fuera de los espacios rurales también es necesario conseguir turno el mismo día llegando temprano por la mañana, incluso esperando desde antes de las 7 am, lo que puede implicar salir de los parajes a las 3 ó 4 de la mañana. El riesgo aquí es no conseguir turno, por la demanda existente y porque los turnos que se dan son limitados. Comenta una agente sanitaria: "las mujeres de mi zona no quieren ir al hospital de Ranchillos [...] porque tienen que ir temprano, a las 4 am. No consiguen número, dan 5 números" (AS, Ranchillos). En Cerro López (Dos Arroyos) una mujer explica: "los turnos tenía que ir bien tempranito a las 5 y media, tenía que agarrar el colectivo que se va a Alem para sacar los turnos y a veces vos llegabas y no había más" (MD). Por su parte, una mujer de la zona rural de Goya plantea la misma dificultad, en este caso agravada por la mayor distancia y la frecuencia limitada del transporte: "tengo que ir a las 3 o 4 am. Acá el remís no te lleva a esa hora, te viene a buscar a las 50 a las 6 , y cuando yo llego [...] a veces no me atienden en el día y tengo que esperar al otro día” (MG). Ante esta situación, la misma mujer termina pagando por la atención en el sistema privado, lo que incluso es más económico para ella, ya que le evita tener que volver otro día o tener que pasar la noche en la ciudad. Así, se observa que el no conseguir turno se articula con la disponibilidad de transporte para llegar al horario esperado, lo que ha sido mencionado como problemático en los tres estudios de caso. No obstante, también parecen existir diferencias entre ellos. En Goya, la gran distancia hace aún más complicado el problema del horario del transporte, lo que se potencia al no existir posibilidad de hacerse controles en las zonas rurales. En Dos Arroyos, la dificultad para los turnos ha sido menos mencionada, posiblemente porque se ha informado la posibilidad de que un familiar de la ciudad saque los turnos, la existencia de profesionales que atienden a embarazadas sin turno o por el hecho de que muchas mujeres dispongan de acceso al subsistema privado por contar con la obra social tabacalera. En Ranchillos si bien la dificultad para los turnos aparece con claridad, también se observa la existencia de iniciativas (no necesariamente exitosas) para lidiar con este problema. En este sentido, se informa que se ha procurado instaurar el turno programado (que se saca días antes de la atención) o la aplicación de la política de que 'las embarazadas no esperan' en algunos servicios.

Para la realización de estudios (sangre, orina y ecografías), en general también es necesaria la obtención de turnos, aunque en este caso son turnos programados. El principal problema aquí es la espera para realizarse los estudios, que puede variar de 1 a 3 meses. Esto se suma a la dificultad de tener que asistir el día y horario del turno, lo que no siempre será posible por limitaciones climáticas (caminos intransitables) o falta de dinero para el transporte: "y al mes la mujer no sabe si va a salir, si va a tener 
la plata, si llovió el camino está feo y no va a poder salir" (PG). Atendiendo a las diferencias entre casos, en Ranchillos se observan iniciativas para reducir la necesidad de turnos, por ejemplo los exámenes de sangre en el hospital de Ranchillos no requieren turno, aunque sólo se hacen los jueves.

Junto al problema de los turnos, también se observan otros de accesibilidad administrativa. Entre ellos se destaca la falta de contrarreferencia, es decir, la falta de devolución de información sobre los tratamientos recibidos o el estado de salud de una paciente que ha sido enviada para atención especializada o para su parto a un centro de atención de mayor complejidad y que decide hacer seguimiento o continuar con la atención en el primer nivel o más cerca de donde vive. En Goya una profesional comenta "la otra dificultad grande es el sistema de referencia y contra referencia" (PG). Un profesional de Ranchillos plantea: "no hay una hoja, que le llamamos de epicrisis, antes de darle el alta, diciendo que ha sido una cesárea, si ha habido alguna complicación o han notado algo en el parto" (PR). En Alem también parece existir el mismo problema, aunque se observan estrategias que podrían contribuir a enfrentarlo como comunicaciones directas del personal médico con agentes sanitarios del territorio para que tengan en cuenta situaciones detectadas.

\section{Estrategias de los profesionales para superar problemas}

Ante estas barreras de accesibilidad, los profesionales en oportunidades ensayan estrategias para disminuir su impacto. Por ejemplo, médicos que tienen alguna capacidad de decisión dentro de un centro asistencial implementan turnos programados o reservan turnos para embarazadas rurales: "se implementó al principio [...] de darles los turnos más o menos programados" (PR). Además, en algunos casos se ha establecido prioridad de atención para que las embarazadas no tengan que esperar. También hay profesionales que suelen utilizar a su favor ciertos mecanismos burocráticos institucionales para conseguir la realización de estudios en el día para pacientes de zonas lejanas, informando situaciones de urgencia que no son tales: "si viene por consultorio externo no le dan turnos, [...] entonces le dan un papelito de ingreso de internación, como que están con pérdidas, [...] es la forma de hacerle las ecografías" (PD). En otras ocasiones, activan contactos personales con profesionales que ocupan cargos decisorios en la institución donde se necesita que se realice la prestación o se organizan ellos mismos para ayudar a las mujeres que viven en zonas más alejadas: "por ejemplo han llegado a las 7 am 0 7.30 y ya no han conseguido turno y están acá cuando yo apenas abro la puerta [...] yo ahí nomás las anoto" (PR). Debe tenerse en cuenta que estas estrategias informales resultan puntuales y precarias puesto que dependen de los recursos relacionales del médico y de su buena voluntad. No obstante, representan una posibilidad de mitigar ciertas dificultades en casos específicos, a la vez que muestran la iniciativa e inventiva de los profesionales.

\section{Síntesis y reflexiones finales}

Antes de continuar, es necesario recordar que los resultados de este trabajo no pueden considerarse representativos de lo que sucede en las provincias en las cuales se realizaron los estudios. No obstante, esto no quita que del estudio puedan derivarse conclusiones y propuestas de utilidad más amplia.

Uno de los aspectos que más se destacan de la investigación es la importancia que juega la distancia geográfica existente entre las mujeres rurales beneficiarias y los efectores del sistema de salud como barrera de accesibilidad. En este sentido, se observan grandes diferencias entre Goya, por un lado, y Dos Arroyos y Ranchillos, por el otro. En primer lugar, en Goya la atención en las zonas rurales está organizada a partir de operativos rurales sin presencia permanente de profesionales ni de agentes sanitarios junto a las comunidades, mientras que en Dos Arroyos y Ranchillos encontramos diferentes CAPS en las zonas rurales que cuentan con profesionales que pueden hacer seguimiento del embarazo y agentes sanitarios que ayudan en la captación de embarazadas. En este aspecto, Ranchillos se destaca por una mayor diversidad y disponibilidad de profesionales en los CAPS que Dos Arroyos. Respecto de la realización de estudios para el seguimiento del embarazo, en los tres casos se observa la necesidad de que las mujeres se trasladen a las zonas urbanas. Aquí los parajes estudiados de 
Goya también quedan ubicados en la peor situación, dada la mayor distancia en kilómetros, la menor disponibilidad de medios de transporte y las distancias a recorrer por caminos de tierra no consolidados. En contraposición, en Ranchillos se observan caminos de tierra más consolidados y la existencia de medios de transporte públicos aún en las zonas rurales. Dos Arroyos se ubica en una posición intermedia, con trayecto de tierra no consolidado para llegar al asfalto y medios de transporte para llegar a la ciudad sólo desde la ruta.

A las barreras geográficas también se suman otras de naturaleza administrativa y económica. A nivel administrativo, en los tres casos se han encontrado dificultades para la obtención de turnos para la realización de estudios, ya que generalmente se requiere traslado previo a las ciudades para solicitar turnos programados que pueden demorar hasta varios meses, lo que se hace aun más difícil en Goya por las mayores distancias. Esto empuja a las mujeres a atenderse en el subsistema privado, más ágil administrativamente pero que requiere de disponer de dinero adicional. En Dos Arroyos esto aparece como menos problemático, ya que muchas mujeres rurales cuentan con cobertura de salud privada por estar vinculadas con el rubro tabacalero. Al mismo tiempo, en todos los casos se observa como barrera administrativa la falta de contra referencia de los niveles de mayor complejidad (ubicados en las ciudades) a los localizados en los territorios (CAPS y operativos rurales), lo que en algunos casos procura ser subsanado a nivel de contactos personales por los profesionales, particularmente en Dos Arroyos, pero no a partir de procedimientos institucionalizados. Por su parte, se observa que los problemas de accesibilidad económica (falta de dinero para pago de transporte o para la realización de estudios en el subsistema privado) se ven agravados por las mayores distancias y la falta de atención a nivel local, como en el caso de Goya y, en general, por los problemas para la obtención de turnos para la realización de estudios.

Finalmente, como aspectos facilitadores de accesibilidad de observan en general la existencia de CAPS en los territorios, la presencia de agentes sanitarios, la disponibilidad de ambulancias para el transporte en caso de emergencias y la existencia de iniciativas informales de los profesionales para resolver los problemas identificados.

De la síntesis anterior se derivan diferentes reflexiones. En primer lugar, se observa que el carácter rural de las poblaciones resulta fundamental a la hora de pensar su acceso a la atención en el ámbito de la salud materna. De esto = sigue la necesidad de políticas públicas que tomen en cuenta la especificidad de estas poblaciones, para generar estrategias que resulten acordes. Incluso, si se acepta la existencia de una desventaja rural, sería oportuno incorporar en las estadísticas el origen rural o urbano de las mujeres que mueren por causas maternas, con el fin de chequear su incidencia.

Segundo, este estudio también ha mostrado que la accesibilidad geográfica no puede considerarse como sinónimo de la distancia de las poblaciones rurales a los centros urbanos, ya que hay variables intermediarias como si los caminos son transitables, la existencia de medios de transporte y la distribución territorial de la atención en salud, que modifican radicalmente su impacto. Así, resulta fundamental que los planificadores no perciban 'lo rural' como homogéneo. No existe una única ruralidad sino diversas, teniéndose que tener en cuenta esta diversidad a la hora de planificar.

Tercero, el estudio mostró la articulación entre diversos tipos de accesibilidad. En efecto, lo geográfico no puede ser pensado sin lo económico, que enmarca la posibilidad de utilizar diversos medios de transporte o de pagar estudios en localidades más cercanas. Tampoco sin lo administrativo, ya que tener que sacar un turno para un estudio un mes antes de realizarlo no tiene las mismas implicaciones para quien tiene que viajar $50 \mathrm{~km}$ (incluyendo parte del trayecto a pie) que para quien solo tiene que tomar un transporte urbano. Así, se observa que las dimensiones de la accesibilidad tienden a funcionar de manera articulada, lo que exige el estudio y la consideración del modo en que ellas se retroalimentan. En la misma línea, resulta fundamental estudiar cómo las barreras administrativas pueden potenciar las desventajas geográficas y económicas. Asumido esto, se hace necesario actuar de manera inteligente para abordar las barreras administrativas con el fin de evitar que sea el mismo sistema sanitario el que se convierta en una fuente básica de desigualdad en salud. 
Focalizando en las diferencias entre los casos, se destaca la existencia de modelos de atención disímiles. Por un lado el caso de Goya, con una atención concentrada en la ciudad, y por el otro el de Ranchillos (en gran medida también el de Dos Arroyos-Alem), que si bien tiene sus dificultades, cuenta con una atención amplia distribuida en el territorio. Las razones de estas diferencias no han sido analizadas. No obstante, resulta evidente la importancia de la distribución territorial de la atención para favorecer la accesibilidad.

Finalmente, nuestro estudio también muestra la existencia de múltiples iniciativas generadas por los actores del sistema de salud para enfrentar las barreras identificadas. Usualmente, las instituciones no suelen ni favorecer la construcción de propuestas para superar estos problemas ni incorporar a las dinámicas institucionales las propuestas generadas espontáneamente por los actores. Esto hace evidente tanto la pérdida de un capital potencial como la limitación y rigidez de estas instituciones. De esto se sigue tanto la necesidad de favorecer espacios locales reflexivos para encontrar alternativas, como la decisión institucional de incorporarlas a las dinámicas institucionales para evaluar su utilidad.

\section{Referencias}

ADAMSON, P. et al. Are marginalized women being left behind?: a population-based study of institutional deliveries in Karnataka, India. $B M C$ Public Health, London, v. 12, n. 30, 2012. Disponível em: <www.biomedcentral.com/1471-2458/12/30 >. Acesso em: 19 dez. 2013.

ARAKAWA, T. et al. Accessibility to tuberculosis treatment: assessment of health service performance. Revista Latino-Americana de Enfermagem, Ribeirão Preto, v. 19, n. 4, p. 9941002, 2011.

BARBASTEFANO, P.; GIRIANELLI, V.; VARGENS, O. $O$ acesso à assistência ao parto para parturientes adolescentes nas maternidades da rede Susa. Revista Gaúcha de Enfermagem, Porto Alegre, v. 31, n. 4, p. 708-714, 2010.

BUCHBINDER, M. Mortalidad infantil y desigualdad socioeconómica en la Argentina: tendencia temporal. Archivos Argentinos de Pediatría, Buenos Aires, v. 106, n. 3, p. 212-218, 2008.

CLEMENTINO, F. S.; MIRANDA, F. N.

Acessibilidade: identificando barreiras na descentralização do controle da tuberculose nas unidades de saúde da família. Revista Enfermagem UERJ, Rio de Janeiro, v. 18, n. 4, p. 584-590, 2010.

COMES, Y.; STOLKINER, A. Si pudiera pagaría: estudio sobre la accesibilidad simbólica de las mujeres usuarias pobres del AMBA a los servicios asistenciales estatales. Anuario de Investigaciones, Buenos Aires, v. 12, p. 137-143, sep. 2005 .

COMES, Y. et al. El concepto de accesibilidad: perspectiva relacional entre población y servicios. Anuario de Investigaciones, Buenos Aires, v. 14, p. 201-209, enero/dic. 2007.

ECHARRI, C. Estratificación socioeconómica y salud materno infantil en México. Papeles de Población, Toluca, v. 10, n. 39, p. 95-128, 2004.

GAIOSO, V.; MISHIMA, S. Satisfacción del usuario en la perspectiva de la aceptabilidad en el escenario de la salud de la familia: abordaje cuali-cuantitativo. Texto, Contexto, Enfermagem, Florianópolis, v. 16, n. 4, p. 617-625, 2007.

GALAMBROS, C. Health care disparities among rural populations: a neglected frontier. Health $\&$ Social Work, Washington, DC, v. 30, n. 3, p. 179181, 2005 .

GRZYBOWSKI, S.; STOLL, K.; KORNELSEN, J. Distance matters: a population based study examining access to maternity services for rural women. BMC Health Services Research, London, v. 11, n. 147, 2011. Disponível em: <www. biomedcentral.com/1472-6963/11/147>. Acesso em: 20 out. 2013.

HARTLEY, M. et al. Depressed mood in pregnancy: prevalence and correlates in two Cape Town periurban settlements. Reproductive Health, London, v. 8, n. 9, 2011. Disponível em: <www.ncbi.nlm.nih. gov/pmc/articles/PMC3113332/>. Acesso em: 19 dez. 2013. 
HENDERSON, S.; KENDALL, E. Culturally and linguistically diverse peoples' knowledge of accessibility and utilization of health services: exploring the need for improvement in health service delivery. Australian Journal of Primary Health, Melbourne, v. 17, n. 2, p. 195-201, 2011.

HOUNTON, S.; BYASS, P.; BRAHIMA, B. Towards reduction of maternal and perinatal mortality in rural Burkina Faso: communities are not empty vessels. Global Health Action, Häggeby, v. 2, maio 2009. Disponível em: <www.ncbi.nlm. nih.gov/pmc/articles/PMC2779943/>. Acesso em: 14 out. 2013 .

HOUWELING, T. et al. Huge poor-rich inequalities in maternity care: an international comparative study of maternity and child care in developing countries. Bulletin of the World Health Organization, Geneva, v. 85, n. 10, p. 745-754, 2007.

IGBERASE, G.; EBEIGBE, P. Maternal mortality in a rural referral hospital in the Niger Delta, Nigeria. Journal of Obstetrics and Gynaecology, London, v. 27, n. 3, p. 275-278, 2007.

IYENGAR, K. et al. Pregnancy-related deaths in Rural Rajasthan, India: exploring causes, context, and care-seeking through verbal autopsy. Journal of Health, Population and Nutrition, Dhaka, v. 27, n. 2, p. 293-302, 2009.

KESTERTON, A. et al. Institutional delivery in rural India: the relative importance of accessibility and economic status. Pregnancy and Childbirth, London, v. 10, n. 30, 2010. Disponível em: <www.biomedcentral.com/1471-2393/10/30 . Acesso em: 20 out. 2013.

LANDINI, F.; COWES, V. G.; D'AMORE, E. Hacia un marco conceptual para repensar la accesibilidad cultural. Cadernos de Saúde Pública, Rio de Janeiro, v. 3o, n. 2, p. 231-244, 2014.

MACIAS, G. Evaluación de la calidad de atención en el centro de salud Campo Verde (San Salvador de Jujuy). Cuadernos de la Facultad de Humanidades y Ciencias Sociales, San Salvador de Jujuy, n. 37, p. 291-305, dic. 2009.

MARTINS, S. et al. Assistance in family health from the perspective of users. Revista Latino-
Americana de Enfermagem, Ribeirão Preto, v. 18, n. 3, p. 436-443, 2010.

MATTHEWS, Z. et al. Examining the "urban advantage" in maternal health care in developing countries. PLOS Medicine, San Francisco, v. 7, n. 9, 2010. Disponível em: <www.plosmedicine. org/article/info\%3Adoi\%2F10.1371\%2Fjournal. pmed.10o0327>. Acesso em: 19 dez. 2013.

MORENO, G. et al. Apreciaciones de la población en condición de desplazamiento forzado sobre los servicios de salud en algunos municipios de Colombia. Revista de la Facultad Nacional de Salud Pública, Medellín, v. 27, n. 2, p. 131-141, 2009.

ONU - ORGANIZACIÓN DE LAS NACIONES UNIDAS. Informe objetivos de desarrollo del milenio. Nueva York, 2010.

PINHO, L. B.; HERNANDEZ, A. B.; KANTORSKI, L. P. Discurso sobre o acolhimiento e a acessibilidade nos servicos comunitarios de saúde mental. Cogitare Enfermagem, Curitiba, v. 14, n. 4, p. 612619, 2009.

RAMÍREZ, J.; HUERTO, P. Objetivos de desarrollo de salud. Revista Tiempo de Paz, Madrid, v. 4, n. 91, p. 47-52, 2008.

RODRÍGUEZ, A. Sobreposición epidemiológica de la malaria y la infección HIV/SIDA y su relación con la salud materno infantil. Ciencia e Investigación Médica Estudiantil Latinoamericana, Lima, v. 10, n. 1, p. 56-64, 2005.

ROJO, M. et al. Reducción de la mortalidad infantil: una experiencia de monitoreo local y sistemático a nivel municipal: Olavarría, provincia de Buenos Aires. Archivos Argentinos de Pediatría, Buenos Aires, v. 104, n. 5, p. 448-453, 2006.

OTALVARO, A. T.; ARANGO, M. C. Accesibilidad de la población habitante de calle a los programas de Promoción y Prevención establecidos por la Resolución 412 de 200o. Investigaciones Andina, Pereira, v. 11, n. 18, p. 23-35, 2009.

TRAVASSOS, C.; MARTINS, M. Uma revisão sobre os conceitos de acesso e utilização de serviços de saúde. Cadernos de Saúde Pública, Rio de Janeiro, v. 20, p. S19o-S198, 2004. Suplemento 2. 
URIBURU, G. et al. Descripción de la salud materno infantil en Argentina: datos comparativos con la ciudad autónoma de Buenos Aires y la Maternidad Sardá (CABA). Revista del Hospital Materno Infantil Ramón Sardá, Buenos Aires, v. 26, n. 4, p. 169-176, 2007.

VARGAS-LORENZO, I.; VAZQUEZ-NAVARRETE, M.; MOGOLLON-PEREZ, A. Acceso a la atención en salud en Colombia. Revista de Salud Pública, Bogotá, v. 12, n. 5, p. 701-712, 2010.

YAKONG, V. et al. Women's experiences of seeking reproductive health care in rural Ghana: challenges for maternal health service utilization. Journal of Advanced Nursing, Oxford, v. 66, n. 11, p. 2431-2441, 2010.

\section{Contribución de los autores}

Todos los autores contribuyeron con el análisis de los datos y aprobaron el manuscrito final.

Recibido: 02/07/2014

Revisado: 13/10/2014

Aprobado: $31 / 10 / 2014$ 